\title{
All inkjet-printed perovskite-based bolometers
}

\author{
Charles Trudeau $\mathbb{I D}^{1,2}$, Patrick Beaupré ${ }^{2}$, Martin Bolduc ${ }^{3}$ and Sylvain G. Cloutier $\mathbb{D}^{1 凶}$
}

We show flexible bolometer devices produced entirely using digital inkjet printing on polymer substrates. The bolometers consist of a silver interdigital electrode thermistor covered with a methylammonium lead trihalide perovskite absorber layer which shows good absorber characteristics at visible wavelengths. Both the standalone thermistor and the complete bolometer devices show polymer PTC thermistor-like behavior over a temperature range of 17 to $36^{\circ} \mathrm{C}$, with a change in resistance up-to six orders of magnitude over this temperature range. The addition of the perovskite absorber to the thermistor structure provides the illumination-dependent behavior proper to bolometers.

npj Flexible Electronics (2020)4:34; https://doi.org/10.1038/s41528-020-00097-2

\section{INTRODUCTION}

Recent advances in ink formulation paved the way towards the use of inkjet printing as a viable alternative for active optoelectronic device fabrication ${ }^{1-6}$. Inkjet printing provides two distinct advantages over more traditional fabrication approaches. As a digital fabrication method, it can allow a dynamic change of the printed pattern architecture with little or no production downtime. It also favors an optimal use of the expensive functional inks, which in turn limits the financial and environmental impacts. With the advent of high-speed manufacturing-scale print engines, inkjet printing is becoming an increasingly appealing fabrication process for low-cost, flexible, active, and passive electronic devices ${ }^{7}$. Smart-buildings, biomedical, and wearable monitoring applications urgently need inexpensive and low-SWAP (size, weight, and power) flexible temperature and light sensors. These critical applications often call for a limited temperature range of operations but require a high level of accuracy and precision. Typical temperature ranges of $30-50$ and $15-40{ }^{\circ} \mathrm{C}$ are usually required for applications in health care/human activity monitoring and for smart building applications, respectively ${ }^{8-12}$. Compatibility with low-cost and low-temperature flexible substrates such as polyethylene terephthalate (PET) and polydimethylsiloxane (PDMS) is also important to boost device performances and/or applicability $^{13-16}$.

The addition of light absorbers to thermistor structures can effectively create bolometer structures capable of sensing light ${ }^{17}$. Combining bolometers and standalone thermistors in a same system can be beneficial for smart building applications, where sensing day/night cycles or overcast conditions can provide additional benefits when compared to standalone temperature sensing ${ }^{18}$. One solution-based family of materials with great promises for light-absorbing capabilities are methylammonium lead trihalide perovskites $\left(\mathrm{CH}_{3} \mathrm{NH}_{3} \mathrm{PbX}{ }_{3}, \mathrm{X}=\mathrm{I}, \mathrm{Cl}\right.$ and/or $\left.\mathrm{Br}\right)$. These precursor-based materials have made tremendous headway in photodetection and energy harvesting applications ${ }^{19-22}$, behaving as outstanding broadband light absorbers ${ }^{23-25}$. In this work, digital inkjet printing is successfully used to produce flexible thermistor and bolometer structures with interdigital silver electrodes and perovskite absorber layers. Both these devices may be easily integrated or fabricated on the same substrate for combined temperature and light sensing.

\section{RESULTS AND DISCUSSION}

Device inner workings

The silver interdigital electrodes are printed, following the thermistor fabrication process depicted in Fig. 1a), using three different pitch parameters $(P)$, namely; 50, 45, and $40 \mu \mathrm{m}$. The width $(W)$ and gap $(G)$ of the interdigital electrodes, depicted in Fig. 1c), are measured and summarized in Supplementary Table 1, along with the pad-to-pad resistance at room temperature. Measurements of $W$ and $G$ are shown in Supplementary Fig. 1. Thickness measurements of the interdigital electrodes are also taken and shown in Supplementary Fig. 2. The pad-to-pad resistance is seen to be highly dependent on $P$. At $P=40 \mu \mathrm{m}$, a pad-to-pad resistance of $10 \pm 5 \Omega$ is measured and suggests shorting between the interdigital electrodes. Indeed, for $P=40 \mu \mathrm{m}$, multiple shorting pathways are observed between the interdigital electrodes, these are shown in Fig. 1d. Line bulging at the ends of the electrode digits occurs due to a known line printing phenomenon and is responsible for the formation of most of these shorting pathways ${ }^{26-28}$. For $P=45 \mu \mathrm{m}$, an average of one electrical shorting pathway is observed per device, while no shorting is observed for the $P=50 \mu \mathrm{m}$ devices. For this reason, only $P=50 \mu \mathrm{m}$ devices are used for bolometer fabrication. We speculate that microscopic electrical pathways are created between the interdigital electrodes in all fabricated devices due to ink micro-splashing while printing. Evidence of ink microsplashing between the interdigital electrodes is highlighted in Fig. 1e. This may explain the measurable pad-to-pad resistance for the $P=50 \mu \mathrm{m}$ interdigital electrodes and the polymer PTC (positive temperature coefficient thermistor) like behavior observed and described in the following sections.

Two important figures of merit for bolometer characterization are the TCR (temperature coefficient of resistance) and the voltage responsivity of the devices. The TCR is the relative change in resistance with temperature and is shown in Eq. 1. The voltage responsivity $\left(R_{\mathrm{v}}\right)$, shown in Eq. 1 , is measured in $\mathrm{VW}^{-1}$, it is the ratio of the output voltage and the incident light flux.

$$
R_{\mathrm{v}}=\frac{\eta a R l_{\mathrm{b}}}{G_{\mathrm{th}}\left(1+\omega^{2} \tau_{\mathrm{th}}^{2}\right)^{0.5}},
$$

Where $\eta$ is the light absorption coefficient of the absorber layer, $R$ is the device resistance, $I_{\mathrm{b}}$ is the bias current, $G_{\mathrm{th}}$ is the thermal 
conductance between the bolometer and its surroundings, $\omega$ is the angular modulation frequency of the incident light, and $\tau_{\text {th }}$ is the thermal response time. This shows that the responsivity of bolometers is proportional to the TCR and the light absorption coefficient. Therefore, a high light absorption coefficient is critical for the absorber layer material in bolometer fabrication ${ }^{29,30}$. This is especially true for flexible bolometers, where thin material layers are wanted for improved device integrity under mechanical stresses occurred during device manipulation ${ }^{31,32}$. The perovskite precursor chosen for the absorber layer provides very high visible light absorption ${ }^{23-25}$, while also providing added humidity stability when compared to other methylammonium lead trihalide perovskite compositions ${ }^{33,34}$.

The absorption of inkjet printed perovskite layers is tested to validate their implementation as light absorbing layers in bolometer structures. A simplified schematic of the reflectance and transmittance measurement set-up is depicted in Supplementary Fig. 3, an integrating sphere is used to capture specular and diffuse reflection, as well as direct and scattered transmission. The absorption spectra of different number of printed perovskite layers are presented in Fig. 2a, while the transmission and reflection spectra are presented in Fig. $2 b$. From these results we observe that a single layer of printed perovskite offers near complete absorption of visible light up to $550 \mathrm{~nm}$, while two printed layers drastically improve absorption up to $750 \mathrm{~nm}$. Further increasing the number of printed layers to three marginally improves absorption at longer wavelengths, while four printed layers offer almost no further improvement. We consider that two



Fig. 1 Printed thermistor and bolometer devices. a Schematic of the printed device fabrication process. b Photograph of completed bolometer device. c Schematic of the silver interdigital electrode with the pitch $(P)$, gap $(G)$ and width $(M)$ parameters. d Photograph of $P=40 \mu \mathrm{m}$ silver interdigital electrodes showing electrical shorting pathways and the line bulging phenomenon (scale bar $=200 \mu \mathrm{m}$ ). e Photograph of interdigital gap showing evidence of silver ink micro-splashing (scale bar $=10 \mu \mathrm{m}$ ). layers of the printed perovskite absorber is enough for visible light sensing to validate bolometer behavior.

\section{Device characterization}

The devices' thermistor behavior is tested and is observed to be stable and repeatable between 17 and $36^{\circ} \mathrm{C}$. Devices with the perovskite light absorbing layer suffer from degradation at temperatures below $17^{\circ} \mathrm{C}$ while under operation. A more detailed description of this degradation is provided in the Supplementary Discussion, photographs of the perovskite undergoing degradation are shown in Supplementary Fig. 4 while electrical abnormalities due to the degradation are shown in Supplementary Fig. 5 . Above $36^{\circ} \mathrm{C}$, both device structures reach their maximum resistance, which no longer fluctuates with increasing temperatures.

The temperature-dependent $I-V$ characteristics of the $P=50 \mu \mathrm{m}$ thermistor and bolometer devices are shown in Fig. $3 a, b$, respectively. The devices' pad-to-pad temperature-dependent resistance, along with the resulting TCR are presented in Fig. 3c. Similar trends in the temperature dependence of the standalone thermistor and of the bolometer devices are observed. The resistance of the devices increases by six orders of magnitude from 17 to $36^{\circ} \mathrm{C}$. This behavior is reminiscent of polymer PTC thermistors, where conductive grains, within a polymer medium, form conductive pathways as the polymer is cooled ${ }^{35,36}$. It is evident from these results that the silver interdigital electrodes on the flexible PET substrate are responsible for the overall temperature dependence of the bolometer devices as both the standalone thermistor and bolometer devices possess almost identical TCR values. Thus, we speculate that the microsplashing of the silver ink is responsible for the formation of increased conductive pathways as the devices are cooled and thus dictate their temperature-dependent behavior.

As shown from Eq. 1, a high TCR is critical for bolometer operations. Bolometer TCR values typically range between $0.1 \%{ }^{\circ} \mathrm{C}^{-1}$ and $9 \%{ }^{\circ} \mathrm{C}^{-1}$ and depend heavily on the absorber material used $^{29,30,37}$. In the case of the devices presented in this article, the change of resistance originates from the silver interdigital electrodes and the flexible PET substrate. This allows for much greater TCR values to be obtained (up to $91 \%{ }^{\circ} \mathrm{C}^{-1}$ ) when compared to typical semiconductor absorber materials.

To validate their use as bolometers the $I-V$ characteristics of both device structures are measured in the dark and under 1 sun illumination, the results are shown in Fig. 4a. The standalone thermistor shows only negligible change in $I-V$ characteristics under illumination, while the bolometer device shows a clear increase in resistance due to the incident light. The devices' resistance response under varying light intensities is also characterized to determine device behavior at lower light levels, the results are presented in Fig. 4b. Once again, the standalone thermistor shows only negligible change in device resistance at any incident light intensity. Interestingly, the bolometer device shows a linear increase in resistance with incident light intensities
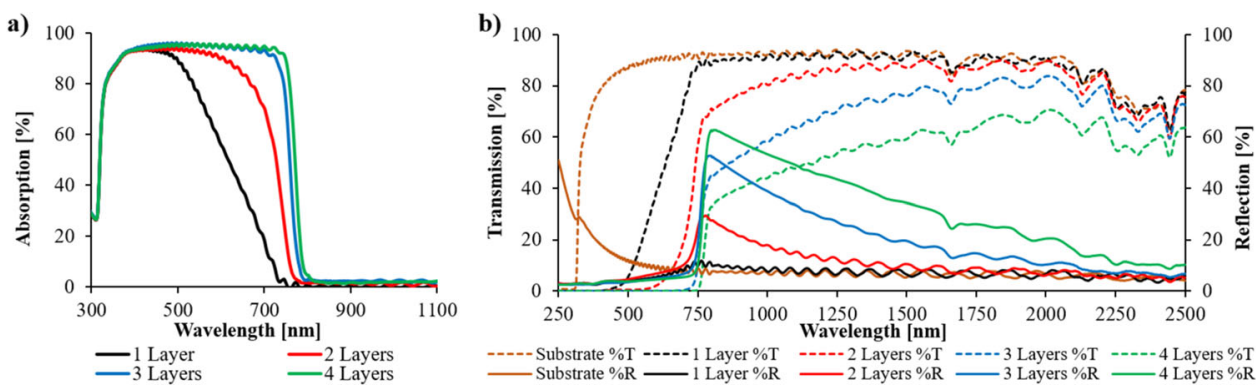

Fig. 2 Optical properties of the printed $\mathrm{CH}_{3} \mathrm{NH}_{3} \mathrm{Pbl}_{3-x} \mathrm{Cl}_{\mathbf{x}}$ perovskite layer. a Absorption spectra of printed $\mathrm{CH}_{3} \mathrm{NH}_{3} \mathrm{Pbl}_{3-x} \mathrm{Cl}_{x}$ perovskite layers. b Transmission and reflection spectra of printed $\mathrm{CH}_{3} \mathrm{NH}_{3} \mathrm{Pbl}_{3-x} \mathrm{Cl}_{x}$ perovskite layers and Novele ${ }^{\mathrm{TM}}$ IJ-220 PET substrates. 

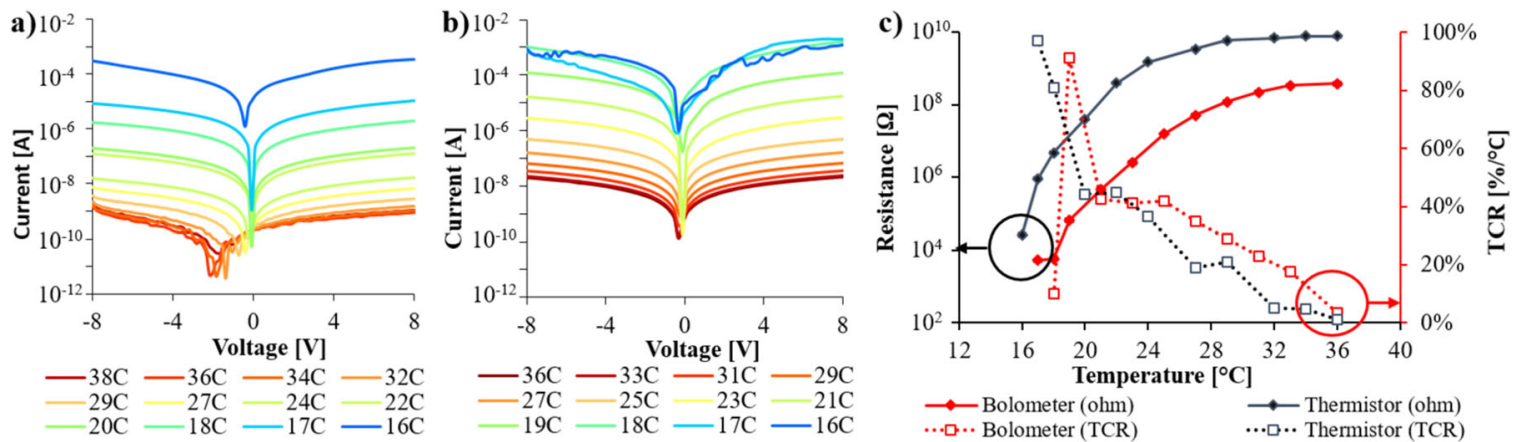

Fig. 3 Temperature-dependent characteristics of the printed thermistor and bolometer devices. a Temperature-dependent I-V characteristics of the $P=50 \mu \mathrm{m}$ thermistor. b Temperature-dependent I-V characteristics of the $P=50 \mu \mathrm{m}$ bolometer. c Temperaturedependent resistance and TCR values of the $P=50 \mu \mathrm{m}$ thermistor and bolometer devices. (error bars representing standard deviation in resistance measurements are present but too small to clearly distinguish).
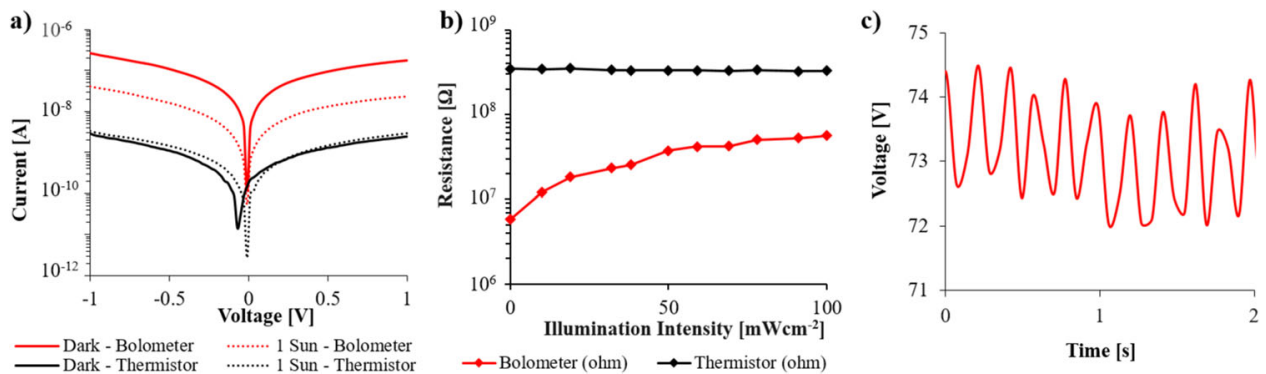

Fig. 4 Light-dependent characteristics of the printed thermistor and bolometer devices. a $I-V$ characteristics of the $P=50 \mu \mathrm{m}$ thermistor and bolometer devices under dark and 1 sun AM1.5 illuminated conditions. b Resistance of the $P=50 \mu m$ thermistor and bolometer devices under varying AM1.5 illumination intensities $\left(I_{\mathrm{b}}=1 \mu \mathrm{A}\right)$. c Transient voltage response of $P=50 \mu \mathrm{m}$ bolometer device under 1 sun AM1.5 illumination modulated at $5 \mathrm{~Hz}\left(I_{\mathrm{b}}=1 \mu \mathrm{A}\right)$.

over the tested range. Moreover, the bolometer devices are shown to respond to light intensities as low as $10 \mathrm{mWcm}^{-2}$. The transient voltage response of the devices is also characterized using 1 sun illumination modulated at $5 \mathrm{~Hz}$, the results are presented in Fig. 4c. The results clearly show a device response from the cyclic illumination. However, this response is an order of magnitude less than observed in the previous on/off illumination tests (Fig. 4a). This transient behavior is more akin to induced thermic effects, where the device has insufficient time to cool down between on/ off cycles, than to direct optoelectric effects. Moreover, since typical perovskite behavior shows decreasing resistance from incident light (due to an influx of photogenerated charges) ${ }^{38,39}$, we deduce that the observed increase in resistance originates from light-induced heating effects.

From the transient voltage results, a $R_{\mathrm{v}}$ of $28.1 \mathrm{VW}^{-1}$ can be calculated (at $5 \mathrm{~Hz}$ ). This $R_{\mathbf{v}}$ value is low when we compared to other flexible bolometer devices, typical values of $>10^{3} \mathrm{VW}^{-1}$ can be found in the literature $29,30,37$. Although our devices possess high light absorption and elevated TCR values, the low $R_{\mathrm{v}}$ value is not surprising. Indeed, thermal conductance between the bolometer and its surroundings $\left(G_{\mathrm{th}}\right)$ is also important in determining the $R_{\mathrm{v}}$. In typical bolometer device architectures, the absorber material is often isolated using a vacuum and/or suspended structures to minimize $G_{\text {th }}$ and thus increase $R_{\mathrm{v}}{ }^{40-43}$. This remains an avenue for further improvement to the printed devices presented.

\section{Device flexibility characterization}

Finally, we characterize the bolometer devices under bending tests to assess their stability and flexibility. Currently, no norms or standards exist for testing flexibility in flexible devices. The most common tests involve cycling bending using a $90^{\circ}$ bending angle ${ }^{32,44}$. Using a custom built cyclic translational stage, cyclic bending tests are performed at 100 bends per minute with a bending radius of $5.5 \mathrm{~mm}$, equating to a bending angle of approximately $90^{\circ}$. A photograph of the bolometer device curved with a bending radius of $5.5 \mathrm{~mm}$ is shown in Fig. 5a, where $L$ is the width of the device, $r$ is the bending radius and $\theta$ is the bending angle. The resistance of the device was measured intermittently after each set of bending cycles, the results are presented in Fig. $5 c$, where $\Delta R$ is the change of resistance from the initial device resistance, $R_{0}$. We observe a modest consistent increase in resistance up to 3000 bending cycles, after which the devices deteriorate rapidly and an abrupt increase in resistance is observed. Major crack formation starts to occur around 3000 bending cycles and after 5000 bending cycles, the devices suffer from complete mechanical failure and are effectively in open circuit states. These cracks spread over multiple interdigital electrodes, and are present in both the perovskite absorber material and the silver electrode material. An example of a crack formed in the devices after 3000 bending cycles is shown in Fig. $5 \mathrm{~b}$.

\section{Lamination and encapsulation}

Device encapsulation/lamination remains to be explored and may offer critical improvements to device mechanical stability under bending stresses and device stability at low temperatures. Lamination can be used to equalize bending stresses and could minimize crack formation thus improving mechanical stability ${ }^{45}$. Lamination can also be used to isolate the device from ambient humidity, and may improve device stability at low temperatures. Conversely, lamination may negatively affect the device responsivity by increasing the total heat capacity of the device, thus decreasing the induced change in temperature from the incident light. We plan to address device lamination while also exploring different absorber materials to optimize device performances and stability in our future works. 

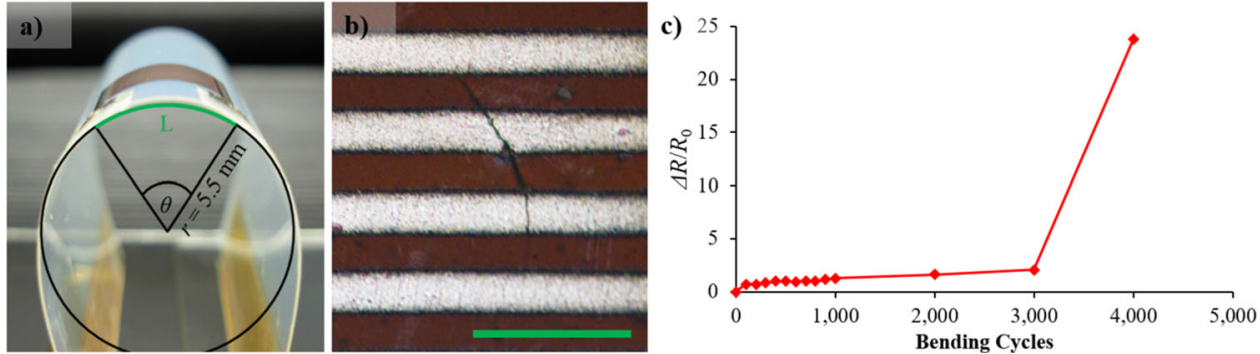

Fig. 5 Characterization of the bolometer devices under bending tests. a Illustration showing $5.5 \mathrm{~mm}$ bending radius used in bending cycle tests. b Photograph of crack formation in the device after 3000 bending cycles. (scale bar $=100 \mu \mathrm{m}$ ) c Change in resistance of the $P=50 \mu \mathrm{m}$ bolometer devices following bending cycles.

\section{METHODS}

\section{Device fabrication}

The devices are printed on flexible Novele $^{\mathrm{TM}}$ IJ-220 PET substrates (Novacentrix) using a Ceradrop F-series inkjet printer. The substrates are chosen for their low cost and high transmittance in the visible range ${ }^{46}$. Two commercial inks are chosen for device fabrication. The DGP 40TE-20C silver nanoparticle ink (ANP) is used for printing the silver interdigital electrode thermistor and the $1101 \mathrm{CH}_{3} \mathrm{NH}_{3} \mathrm{Pbl}_{3-\mathrm{x}} \mathrm{Cl}_{\mathrm{x}}$ (with $x$ « 1) perovskite precursor (Ossila) is used for printing the perovskite absorber layer.

The $8.5^{\prime \prime} \times 11.5^{\prime \prime} \times 0.14 \mathrm{~mm}$ sheets of Novele ${ }^{\mathrm{TM}} \mathrm{IJ}-220$ PET substrates are cut into $15 \mathrm{~cm} \times 10 \mathrm{~cm}$ rectangles for printing purposes. The cut substrates are placed on the printer platen which holds them through a vacuum. The substrates are cleaned to remove particles by blowing filtered compressed nitrogen and by wiping with a dry non-abrasive lint-free clean room wipe.

Prior to printing, the ANP DGP 40TE-20C silver nanoparticle ink is filtered through a $0.45 \mu \mathrm{m}$ PTFE (Polytetrafluoroethylene) filter prior to filling a $1 \mathrm{pL}$ Dimatix inkjet cartridge. The 1101 perovskite ink is heated to $70^{\circ} \mathrm{C}$ for $2 \mathrm{~h}$ and cooled to room temperature to allow for the complete re-dissolution of the solutes, as specified by the manufacturer. The ink is then filtered through a $0.45 \mu \mathrm{m}$ PTFE filter prior to filling a $10 \mathrm{pL}$ Dimatix inkjet cartridge.

The Dimatix inkjet cartridges filled with the two ink solutions are mounted into a Ceradrop F-Series inkjet printer. The printing platen is heated to $60^{\circ} \mathrm{C}$ and the interdigital electrodes are printed using a single nozzle in vector mode printing with a drop separation distance of $17 \mu \mathrm{m}$. Once the electrodes are printed, a short optical annealing treatment is performed using the printer's built-in near-infrared (NIR) dryer module (Adphos ${ }^{\circledR}$ CER-42-250) operated at $60 \%$ intensity with a platen displacement speed of $5 \mathrm{mms}^{-1}$. This optical treatment solidifies the ink and prepares the sample for the following oven annealing step. The substrate with the optically annealed electrodes is anchored to a dummy glass substrate and thermally annealed in an oven at $180^{\circ} \mathrm{C}$ for $1 \mathrm{~h}$. This process is found to be necessary to avoid damaging the PET substrates while heating to temperatures above $120^{\circ} \mathrm{C}$. This thermal annealing temperature is optimized to minimized electrode resistance, as shown in Supplementary Fig. 6.

To print the absorbing layer, the substrates with the annealed interdigital electrodes are placed on the printer platen, and the platen temperature is brought down to $30^{\circ} \mathrm{C}$ in preparation for perovskite printing. Two layers of perovskite ink are printed in an $8 \mathrm{~mm} \times 8 \mathrm{~mm}$ square pattern atop the silver interdigital electrodes using a drop separation distance of $25 \mu \mathrm{m}$. Once the perovskite light absorbing layer is printed, a short optical annealing treatment is performed using the NIR dryer module operating at $5 \%$ intensity with a platen displacement speed of $20 \mathrm{mms}^{-1}$. A further oven annealing step is performed at $90^{\circ} \mathrm{C}$ for $3 \mathrm{~h}$ to ensure conversion to a crystalline perovskite layer ${ }^{1}$, followed by rapid cooling by placing the substrates on a heat sink. All printing processes are performed at atmospheric conditions with noncontrolled relative humidity of $25-45 \%$ using a Ceradrop F-Series printer.

The device fabrication process is depicted in Fig. 1a, while a photograph of a completed bolometer device is shown in Fig. $1 \mathrm{~b}$, and a design schematic of the silver interdigital electrode identifying key device parameters is shown in Fig. 1c.

\section{Characterization}

Printed device feature sizes are measured using the visible camera and image processing software on the $F$ series printer. Electrical characterization is performed using a Keithley 2400 SMU. Temperature control for thermistor behavior characterization is performed using a 1487-1013-ND
(Laird Technologies) thermoelectric cooler (TEC) and a k-type thermocouple. TCR calculations are performed using Eq. 2,

$a(T)=100 \cdot \frac{1}{R(T)} \cdot \frac{\mathrm{d} R(T)}{\mathrm{d} T}$,

where $a(T)$ is the temperature dependent TCR in $\%{ }^{\circ} \mathrm{C}^{-1}, R(T)$ is the temperature dependent pad-to-pad resistance in $\Omega$, and $T$ is the temperature in ${ }^{\circ} \mathrm{C}$.

Absorption spectra are calculated from transmission and reflection spectra using Eq. 3,

$A(\lambda)=100-[R(\lambda)+T(\lambda)]$,

where $A(\lambda)$ is the wavelength dependent absorption in $\%, R(\lambda)$ is the wavelength dependent reflection in $\%$ and $T(\lambda)$ is the wavelength dependent transmission in \%. The transmission and reflection spectra are acquired using a Carry 7000 spectrophotometer (Agilent) with an integrating sphere to capture specular and diffuse reflection, as well as direct and scattered transmission.

A solar simulator (Oriel Instruments 91160-1000) with a $1000 \mathrm{~W}$ Xenon lamp is used as an illumination source for bolometer behavior validation. To characterize device behavior under cyclic bending stress, a custom cyclic translational stage was built to accommodate the devices. Cyclic bending was performed at 100 bends per minute with a bending radius of $5.5 \mathrm{~mm}$, equating to a bending angle of approximately $90^{\circ}$. Additional device characterization details are provided in the Supplementary Method.

\section{DATA AVAILABILITY}

The data that support the findings of this study are available from the authors on reasonable request.

\section{CODE AVAILABILITY}

The current study makes no use of custom code or mathematical algorithm that is deemed central to the conclusions presented.

Received: 6 May 2020; Accepted: 20 November 2020;

Published online: 11 December 2020

\section{REFERENCES}

1. Trudeau, C. et al. Inkjet-printing of methylammonium lead trihalide perovskite as active layers for optoelectronic devices. MRS Adv. 3, 1-6 (2018).

2. Bernacka-Wojcik, I., Wojcik, P. J., Aguas, H., Fortunato, E. \& Martins, R. Inkjet printed highly porous $\mathrm{TiO} 2$ films for improved electrical properties of photoanode. J. Colloid Interf. Sci. 465, 208-214 (2016).

3. Mathies, F. et al. Multipass inkjet printed planar methylammonium lead iodide perovskite solar cells. J. Mater. Chem. A 4, 19207-19213 (2016).

4. Hashmi, S. G. et al. Long term stability of air processed inkjet infiltrated carbonbased printed perovskite solar cells under intense ultra-violet light soaking. J. Mater. Chem. A 5, 4797-4802 (2017).

5. Eggenhuisen, T. M. et al. High efficiency, fully inkjet printed organic solar cells with freedom of design. J. Mater. Chem. A 3, 7255-7262 (2015).

6. Yang, W. S. et al. lodide management in formamidinium-lead-halide-based perovskite layers for efficient solar cells. Science 356, 1376-1379 (2017). 
7. Leenen, M. A., Arning, V., Thiem, H., Steiger, J. \& Anselmann, R. Printable electronics: flexibility for the future. Phys. Status Solidi 206, 588-597 (2009).

8. Vaz, A. et al. Full passive UHF tag with a temperature sensor suitable for human body temperature monitoring. IEEE Trans. Circuits Syst. II 57, 95-99 (2010).

9. Kokkoris, C. P. et al. Long-term ambulatory temperature monitoring in a subject with a hypernychthemeral sleep-wake cycle disturbance. Sleep 1, 177-190 (1978).

10. Lim, C. L., Byrne, C. \& Lee, J. K. Human thermoregulation and measurement of body temperature in exercise and clinical settings. Ann. Acad. Med. Singap. 37, 347 (2008).

11. Uribe, O. H., Martin, J. P. S., Garcia-Alegre, M. C., Santos, M. \& Guinea, D. Smart building: decision-making architecture for thermal energy management. Sensors 15, 27543-27568 (2015).

12. Moreno, M. V., Skarmeta, A. F., Venturi, A., Schmidt, M., \& Schuelke, A., Context sensitive indoor temperature forecast for energy efficient operation of smart buildings. 2015 IEEE 2nd World Forum on Internet of Things (WF-IoT), 705-710 (IEEE, Milan, 2015).

13. Bessonov, A. A. \& Kirikova, M. N. Flexible and printable sensors. Nanotechnol. Russ. 10, 165-180 (2015)

14. Yan, C., Wang, J. \& Lee, P. S. Stretchable graphene thermistor with tunable thermal index. ACS Nano 9, 2130-2137 (2015).

15. Li, Y., Samad, Y. A. \& Liao, K. From cotton to wearable pressure sensor. J. Mater. Chem. A 3, 2181-2187 (2015).

16. Wang, $X$. et al. Dynamic pressure mapping of personalized handwriting by a flexible sensor matrix based on the mechanoluminescence process. Adv. Mater. 27, 2324-2331 (2015).

17. Van Hoof, C., De Moor, P. Handbook of Infrared Detection Technologies Ch. 12 (Elsevier Science Publisher, Amsterdam, 2002).

18. Morón, C., Payán, A., García, A. \& Bosquet, F. Domotics project housing block. Sensors 16, 741 (2016).

19. Fang, Y., Dong, Q., Shao, Y., Yuan, Y. \& Huang, J. Highly narrowband perovskite single-crystal photodetectors enabled by surface-charge recombination. Nat. Photonics 9, 679-686 (2015).

20. Saidaminov, M. I. et al. Planar-integrated single-crystalline perovskite photodetectors. Nat. Commun. 6, 8724 (2015)

21. Ka, I., Gerlein, L. F., Nechache, R. \& Cloutier, S. G. High-performance nanotubeenhanced perovskite photodetectors. Sci. Rep. 7, 45543 (2017).

22. Noh, J. H., Im, S. H., Heo, J. H., Mandal, T. N. \& Seok, S. I. Chemical management for colorful, efficient, and stable inorganic-organic hybrid nanostructured solar cells. Nano Lett. 13, 1764-1769 (2013).

23. Zhao, X. G. et al. Cu-In halide perovskite solar absorbers. J. Am. Chem. Soc. 139, 6718-6725 (2017).

24. Gao, P., Grätzel, M. \& Nazeeruddin, M. K. Organohalide lead perovskites for photovoltaic applications. Energy Environ. Sci. 7, 2448-2463 (2014).

25. Yin, W. J., Yang, J. H., Kang, J., Yan, Y. \& Wei, S. H. Halide perovskite materials for solar cells: a theoretical review. J. Mater. Chem. A 3, 8926-8942 (2015).

26. Soltman, D. \& Subramanian, V. Inkjet-printed line morphologies and temperature control of the coffee ring effect. Langmuir 24, 2224-2231 (2008).

27. Derby, B. Inkjet printing of functional and structural materials: fluid property requirements, feature stability, and resolution. Annu. Rev. Mater. Res. 40, 395-414 (2010).

28. Kwon, J. S., Lee, D. \& Oh, J. Formation and characterization of inkjet-printed nanosilver lines on plasma-treated glass substrates. Appl. Sci. 8, 280 (2018).

29. Hyseni, G., Caka, N. \& Hyseni, K. Infrared thermal detectors parameters: semiconductor bolometers versus pyroelectrics. WSEAS Trans. Circuits Syst. 9, 238-247 (2010).

30. Dayeh, S. A., Butler, D. P., Celik-Butler, Z. \& Wisian-Neilson, P. Uncooled micromachined bolometer arrays on flexible substrates. SPIE 5074, 537-547 (2003).

31. Li, H., Tang, Z., Liu, Z. \& Zhi, C. Evaluating flexibility and wearability of flexible energy storage devices. Joule 3, 613-619 (2019).

32. Harris, K. D., Elias, A. L. \& Chung, H. J. Flexible electronics under strain: a review of mechanical characterization and durability enhancement strategies. J. Mater. Sci. 51, 2771-2805 (2016).

33. Li, F. \& Liu, M. Recent efficient strategies for improving the moisture stability of perovskite solar cells. J. Mater. Chem. A 5, 15447-15459 (2017).

34. Lee, M. M., Teuscher, J., Miyasaka, T., Murakami, T. N. \& Snaith, H. J. Efficient hybrid solar cells based on meso-superstructured organometal halide perovskites. Science 338, 643-647 (2012).

35. Zeng, Y. et al. Positive temperature coefficient thermistors based on carbon nanotube/polymer composites. Sci. Rep. 4, 6684 (2014).

36. Struempler, R. G. Polymer composites for temperature and current sensors. SPIE 2779, 377-382 (1996)

37. Voshell, A., Dhar, N. \& Rana, M. M. Materials for microbolometers: vanadium oxide or silicon derivatives. SPIE 10209, 102090M (2017).
38. Takshi, A., Bebeau, J. \& Rahimi, F. Perovskite based photosensor for electrochemical studies. SPIE 9944, 99440R (2016).

39. Yang, Z., Dou, J. \& Wang, M. Two Dimensional Materials for Photodetector (Inte chOpen, London, 2018)

40. Niklaus, F. et al. Uncooled infrared bolometer arrays operating in a low to medium vacuum atmosphere: performance model and tradeoffs. SPIE $\mathbf{6 5 4 2}$ $65421 \mathrm{M}(2007)$

41. Eriksson, P., Andersson, J. Y. \& Stemme, G. Thermal characterization of surfacemicromachined silicon nitride membranes for thermal infrared detectors. J. Microelectromech. Syst. 6, 55-61 (1997).

42. Aliane, A. et al. Mechanical modeling and characterization of suspended cooled silicon bolometers for sub-millimeter and millimeter waves polarization detection. Sens. Actuat. A 296, 254-264 (2019).

43. Agnese, P., Buzzi, C., Rey, P., Rodriguez, L. \& Tissot, J. L. New technological development for far-infrared bolometer arrays. SPIE 3698, 284-290 (1999).

44. de Mulatier, S., Ramuz, M., Coulon, D., Blayac, S. \& Delattre, R. Mechanical characterization of soft substrates for wearable and washable electronic systems. APL Mater. 7, 031505 (2019).

45. Park, M. H. et al. Flexible lamination encapsulation. Adv. Mater. 27, 4308-4314 (2015).

46. Zardetto, V., Brown, T. M., Reale, A. \& Di Carlo, A. Substrates for flexible electronics: a practical investigation on the electrical, film flexibility, optical, temperature, and solvent resistance properties. J. Polym. Sci. Pol. Phys. 49, 638-648 (2011).

\section{ACKNOWLEDGEMENTS}

C.T. thanks the National Optics Institute (INO) in Quebec, Canada, for support via INO's scholarship of excellence awards. S.G.C. also thanks the Canada Research Chairs and the NSERC Discovery programs for their support.

\section{AUTHOR CONTRIBUTIONS}

C.T. and M.B. are responsible for device designs. C.T. developed the fabrication process and fabricated the devices. C.T. and P.B. performed device characterization. C.T., M.B., and S.G.C. are responsible for experimental design. C.T. wrote the paper, with editing contributions from all authors.

\section{COMPETING INTERESTS}

The authors declare no competing interests.

\section{ADDITIONAL INFORMATION}

Supplementary information is available for this paper at https://doi.org/10.1038/ s41528-020-00097-2.

Correspondence and requests for materials should be addressed to S.G.C.

Reprints and permission information is available at http://www.nature.com/ reprints

Publisher's note Springer Nature remains neutral with regard to jurisdictional claims in published maps and institutional affiliations.

\begin{abstract}
Open Access This article is licensed under a Creative Commons Attribution 4.0 International License, which permits use, sharing, adaptation, distribution and reproduction in any medium or format, as long as you give appropriate credit to the original author(s) and the source, provide a link to the Creative Commons license, and indicate if changes were made. The images or other third party material in this article are included in the article's Creative Commons license, unless indicated otherwise in a credit line to the material. If material is not included in the article's Creative Commons license and your intended use is not permitted by statutory regulation or exceeds the permitted use, you will need to obtain permission directly from the copyright holder. To view a copy of this license, visit http://creativecommons. org/licenses/by/4.0/.
\end{abstract}

(c) The Author(s) 2020 